\title{
Evaluation of maternal rhesus blood type as a risk factor in adverse pregnancy outcomes in Korea: a nationwide health insurance database study
}

Yihua Jin, MD, Meari Dong, MD', Seung Woo Yang, MD, PhD¹, Kyu-Min Lee, BS², Sung Won Han, PhD², Shin Hee Seo, MD¹, Ajin Lee, MD'1, In Sook Sohn, MD, PhD', Han Sung Kwon, MD, PhD', Geum Joon Cho, MD, PhD ${ }^{3}$, Han Sung Hwang, MD, PhD

\footnotetext{
${ }^{1}$ Division of Maternal and Fetal Medicine, Department of Obstetrics and Gynecology, Research Institute of Medical Science, Konkuk University School of Medicine; ${ }^{2}$ School of Industrial Management Engineering, Korea University; ${ }^{3}$ Department of Obstetrics and Gynecology, Korea University College of Medicine, Seoul, Korea
}

\section{Objectives}

The current study aimed to investigate whether pregnancy outcomes are affected by maternal rhesus (Rh) status by comparing the primigravida pregnancy outcomes of Rh-negative women with those of Rh-positive women.

\section{Methods}

The study data were collected from the Korea National Health Insurance Claims Database and the National Health Screening Program for Infants and Children. In total, 1,664,882 primigravida women who gave birth between January 1, 2007 and December 31, 2014, were enrolled in this study. As the risk and severity of sensitization response increases with each subsequent pregnancy, only primigravida women were enrolled. The patients were divided into 2 groups according to Rh status, and the pregnancy outcomes were compared.

\section{Results}

In total, 1,661,320 women in the Rh-positive group and 3,290 in the Rh-negative group were assessed. With regard to adverse pregnancy outcomes, there was no statistically significant difference between the 2 groups in terms of the prevalence of preeclampsia, postpartum hemorrhage, abruptio placenta, placenta previa, and uterine artery embolization. A univariate analysis revealed that none of the adverse pregnancy outcomes were significantly correlated to Rh status (preeclampsia: odds ratio [OR], 1.00, 95\% confidence interval $[\mathrm{Cl}], 0.81-1.23$; postpartum hemorrhage: OR, 1.10, 95\% Cl, 0.98-1.24; abruptio placenta: OR, 0.80, 95\% Cl, 0.46-1.37; and placenta previa: OR, 1.08, $95 \% \mathrm{Cl}, 0.78-1.42)$. The adjusted ORs of postpartum hemorrhage and preterm birth did not significantly differ.

\section{Conclusion}

Maternal Rh status is not associated with adverse outcomes in primigravida women.

Keywords: Rhesus blood-group system; Pregnancy outcomes; Gravidity; National health programs

Received: 2019.12.28. Revised: 2020.02.14. Accepted: 2020.03.04.

Corresponding author: Han-Sung Hwang, MD, PhD

Department of Obstetrics and Gynecology, Konkuk University

School of Medicine, 120 Neungdong-ro, Gwangjin-gu, Seoul

05029, Korea

E-mail: hwanghs@kuh.ac.kr

https://orcid.org/0000-0003-0622-0701

\author{
Geum Joon Cho, MD, $\mathrm{PhD}$ \\ Department of Obstetrics and Gynecology, Korea University \\ College of Medicine, 148 Gurodong-ro, Guro-gu, Seoul 08308, \\ Korea \\ E-mail: md_cho@hanmail.net \\ https://orcid.org/0000-0001-6761-0944
}

Articles published in Obstet Gynecol Sci are open-access, distributed under the terms of the Creative Commons Attribution Non-Commercial License (http://creativecommons. org/licenses/by-nc/3.0/) which permits unrestricted non-commercial use, distribution, and reproduction in any medium, provided the original work is properly cited.

Copyright (C) 2020 Korean Society of Obstetrics and Gynecology 


\section{Obstetrics \& Gynecology Science}

Yihua Jin, et al. Rhesus blood type and pregnancy outcome

\section{Introduction}

A person's blood type is determined according to the specific antigen types on the erythrocyte membranes of red blood cells (RBCS). The 2 main factors that determine the blood type are $A B O(A, B, A B$, and $O)$ and rhesus (Rh) (positive or negative). Karl Landsteiner first discovered the $A B O$ blood group system in 1900 when he was investigating the causes of some fatal transfusions [1]. The ABO blood group system was named according to the different agglutinins, or blood group antigens, including $\mathrm{A}, \mathrm{B}$, and $\mathrm{H}$ (or $\mathrm{O}$ ) antigens, on the surface of human RBCs [1]. Based on the presence or absence of the Rh factor, the blood group system is called the Rh blood group system. Rh represents the first 2 letters of the name Macacus Rhesus. In 1940, during animal experiments, Landsteiner and other scientists discovered that rhesus monkeys and most human RBCs have antigenic Rh blood types, and this was used in naming the system [2]. With continuous studies of the Rh blood groups, the Rh blood group system was found to be the most complex system in the RBCs [3]. The discovery of the Rh blood type has played an important role not only in guiding blood transfusions more scientifically but also in improving experimental diagnoses and clinical immunotherapy.

The blood type may affect human health and diseases with a wide range of expression in human cells and tissues, including platelets, epithelium, and vascular endothelium [4,5]. Therefore, several studies showed the clinical significance of the biological characteristics of the ABO blood system, particularly with regard to cancer, cardiovascular disease, and pregnancy-related disease [6-8]. Numerous reports revealed that the $\mathrm{ABO}$ blood group may be associated with some risk factors for unfavorable pregnancy outcomes [9].

However, data about whether maternal Rh blood type alone, without consideration of alloimmune sensitization, is associated with the development of pregnancy-related diseases are limited. Moreover, the Rh-negative population is extremely small. Thus, a large population must be evaluated to assess the role of Rh blood type in identifying women at risk of developing pregnancy-related complications.

Thus, this study aimed to investigate whether pregnancy outcomes are affected by maternal Rh status by comparing the primigravida pregnancy outcomes of Rh-negative women with those of Rh-positive women in a nationwide population study.

\section{Materials and methods}

\section{Health care in Korea}

Approximately $97 \%$ of the Korean population is enrolled in the Korea National Health Insurance $(\mathrm{KNHI})$ program. All claims data are stored in the $\mathrm{KNHI}$ claims database. As part of the KNHI system, a National Health Screening Program for Infants and Children (NHSP-IC) was started in 2007, and it includes information about physical examination findings, anthropometric measurements, and developmental screening results after birth. This study used information from the $\mathrm{KNHI}$ claims database to identify all women who gave birth between January 1, 2007 and December 31, 2014. Moreover, whether these women were Rh-positive or Rh-negative based on the applicable codes from the International Classification of Disease, 10th Revision was assessed. The data of women who met the inclusion criteria were linked to those of their offspring in the NHSP-IC database.

\section{Dataset and outcomes}

Fig. 1 shows the flowchart of participant enrollment. Using the KNHI claims data, we identified all women who gave birth between January 1, 2007 and December 31, $2014(n=3,383,282)$. The maternal datasets were merged with those in the NHSP-IC database. Multigravida women $(n=1,650,500)$, those whose offspring had not undergone

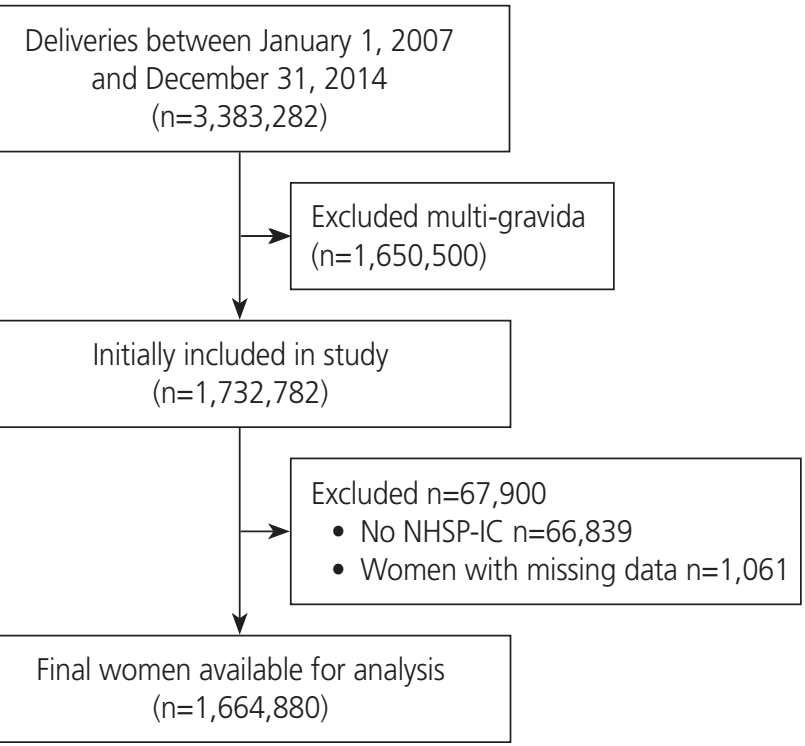

Fig. 1. Flowchart of participant enrollment. NHSP-IC, National Health Screening Program for Infants and Children. 


\section{Obstetrics \& Gynecology Science}

Vol. 63, No. 4, 2020

NHSP-IC health examinations $(n=66,839)$, and those with missing data $(n=1,061)$ were excluded from the study. Data regarding maternal and offspring outcomes were extracted. The information included pregnancy outcomes, such as parity, type of delivery, pulmonary embolism, postpartum hemorrhage, abruptio placenta, placenta previa, and uterine artery embolization. Furthermore, the characteristics of the neonates, such as sex, whether delivered preterm, and birth weight, were evaluated. Preterm birth was defined as birth at a gestational age $<37$ weeks, low birth weight (LBW) as birth weight $<2.5 \mathrm{~kg}$, and large for gestational age (LGA) as birthweight $>4.0 \mathrm{~kg}$.

\section{Statistical analysis}

Continuous and categorical variables were expressed as mean \pm standard deviation and number (percentage), respectively. The clinical characteristics of the participants were compared using $t$-test for continuous variables and the $\chi^{2}$ test for categorical variables. The risks of Rh-negative blood type were evaluated via multiple regression analyses. The risk of postpartum hemorrhage and preterm birth was adjusted for each related risk factor (postpartum hemorrhage: age, multiple pregnancy, cesarean section, preterm birth, LBW, LGA, preeclampsia, abruptio placenta, and placenta previa; preterm birth: age, multiple pregnancy, and preeclampsia). All tests were 2 -sided, and a $P$-value $<0.05$ was considered statistically significant. Statistical analyses were performed using SAS for Windows (version 9.4; SAS, Cary, NC, USA).

\section{Results}

\section{Baseline characteristics of the study population}

The clinical characteristics of the participants are shown in Table 1. There were no significant differences in terms of age at delivery and multiple pregnancy and cesarean sec-

Table 1. Clinical characteristics of the participants

\begin{tabular}{|c|c|c|c|}
\hline Characteristics & Rh-positive ( $n=1,661,320)$ & Rh-negative $(n=3,290)$ & $P$-value \\
\hline Age (yr) & $29.8 \pm 3.7$ & $29.7 \pm 3.5$ & 0.444 \\
\hline Age $>35$ yr at delivery & $161,506(9.7)$ & $317(8.8)$ & 0.058 \\
\hline Multiple gestation & $33,479(2.0)$ & $82(2.3)$ & 0.270 \\
\hline Cesarean section & $617,044(37.1)$ & $1,311(36.4)$ & 0.323 \\
\hline Preeclampsia & $40,998(2.5)$ & $89(2.5)$ & 0.998 \\
\hline Postpartum hemorrhage & $130,422(7.9)$ & $308(8.5)$ & 0.124 \\
\hline Placenta abruption & $7,528(0.5)$ & $13(0.4)$ & 0.407 \\
\hline Placenta previa & $18,899(1.1)$ & $43(1.2)$ & 0.757 \\
\hline Uterine artery embolization & $2,101(0.1)$ & $6(0.2)$ & 0.501 \\
\hline
\end{tabular}

Data were represented as mean \pm standard deviation and number (\%).

Rh, rhesus.

Table 2. Neonatal weight differences between the rhesus (Rh)-positive and Rh-negative groups

\begin{tabular}{lccc}
\hline Characteristics & Rh-positive $(\mathbf{n = 1 , 6 6 1 , 3 2 0 )}$ & Rh-negative $(\mathbf{n}=\mathbf{3 , 2 9 0 )}$ & $\boldsymbol{P}$-value \\
\hline Male sex & $853,655(51.38)$ & $1,883(52.20)$ & 0.325 \\
Preterm birth & $57,406(3.46)$ & $129(3.58)$ & 0.691 \\
Neonatal weight (g) & $3.18 \pm 0.49$ & $3.17 \pm 0.52$ & 0.301 \\
Normal weight & $1,520,122(91.50)$ & $3,316(91.93)$ & 0.273 \\
Low birth weight & $82,309(4.95)$ & $181(5.02)$ & 0.221 \\
Large birth weight & $58,889(3.54)$ & $110(3.05)$ & 0.313 \\
\hline
\end{tabular}

Data were presented as mean \pm standard deviation and number (\%). Low birth weight: $<2,500 \mathrm{~g}$, large birth weight: $>4,000 \mathrm{~g}$, and preterm delivery: $<37$ gestational weeks. 


\section{Obstetrics \& Gynecology Science}

Yihua Jin, et al. Rhesus blood type and pregnancy outcome

tion rates between the Rh-positive group $(n=1,661,320)$ and Rh-negative group $(n=3,290)$. Moreover, adverse delivery outcomes did not significantly differ between the 2 groups. Moreover, preterm birth rates and birth weight (corrected for gestational age) did not significantly differ between the Rhpositive and Rh-negative groups (Table 2).

\section{Univariate regression analysis of $\mathbf{R h}$ blood type as a risk factor for pregnancy outcomes}

Univariate regression analyses were performed to evaluate the associations between Rh blood type and various clinical outcomes (Table 3). The Rh blood type was not a risk factor for preterm birth (odds ratio [OR], 1.13, 95\% confidence interval [Cl], 0.91-1.41), LBW (OR, 1.01, 95\% Cl, 0.87-1.18), and LGA (OR, 0.86, 95\% Cl, 0.71-1.04). Rh blood type was not a risk factor for adverse pregnancy outcomes (preeclampsia: $O R, 1.00,95 \% \mathrm{Cl}, 0.81-1.23$; postpartum hemorrhage: OR, 1.10, 95\% Cl, 0.98-1.24; abruptio placenta: OR, 0.80, 95\% Cl, 0.46-1.37; and placenta previa: OR, 1.08, 95\% Cl, 0.78-1.42). Moreover, age-adjusted univariate regression analyses revealed no significant relationships between the $\mathrm{Rh}$ blood type and these factors.

\section{Multiple regression analysis of rhesus blood type as a risk factor for postpartum hemorrhage and preterm birth}

Based on the univariate analysis, the adjusted ORs for postpartum hemorrhage and preterm birth were analyzed be-

Table 3. Univariate regression analysis of rhesus blood type as a risk factor of pregnancy outcomes

\begin{tabular}{lcc}
\hline Characteristics & OR (95\% CI) & $\begin{array}{c}\text { Age-adjusted } \\
\text { OR }(95 \% \text { Cl) }\end{array}$ \\
\hline Multiple pregnancy & $1.13(0.91-1.41)$ & $1.15(0.92-1.43)$ \\
Cesarean section & $0.97(0.90-1.03)$ & $0.97(0.91-1.04)$ \\
Preterm birth & $1.04(0.87-1.24)$ & $1.04(0.87-1.24)$ \\
LBW & $1.01(0.87-1.18)$ & $1.02(0.88-1.18)$ \\
LGA & $0.86(0.71-1.04)$ & $0.86(0.71-1.04)$ \\
Preeclampsia & $1.00(0.81-1.23)$ & $1.00(0.81-1.24)$ \\
Postpartum hemorrhage & $1.10(0.98-1.24)$ & $1.10(0.98-1.23)$ \\
Placental abruption & $0.80(0.46-1.37)$ & $0.80(0.46-1.38)$ \\
Placental previa & $1.08(0.78-1.42)$ & $1.07(0.79-1.44)$ \\
\hline
\end{tabular}

$\mathrm{OR}$, odds ratio; $\mathrm{Cl}$, confidence interval; LBW, low birth weight; LGA, large for gestational age. cause there was no significant relationship between Rh blood type and clinical outcomes (Table 4). The risk factors for postpartum hemorrhage were age, multiple pregnancy, cesarean section, preterm birth, LBW, LGA, preeclampsia, abruptio placenta, and placenta previa. Preterm birth was adjusted for age, multiple pregnancy, and preeclampsia. After adjusting for the factors, no relationships were found between $\mathrm{Rh}$ blood type and postpartum hemorrhage $(\mathrm{OR}, 1.10,95 \% \mathrm{Cl}$, 0.98-1.23) and preterm birth (OR, $0.02,95 \% \mathrm{Cl}, 0.85-1.23)$.

\section{Discussion}

The proportions of blood type differ according to race, region, and ethnicity. In general, Africa, the Middle East, Europe, India, and Central Asia have higher Rh-negative blood rates than other regions. In Korea, Rh-negative is considered a rare blood type according to the Korean Red Cross standards, with an incidence of $0.4 \%$; however, pregnancy can lead to several complications among Rh-negative women [10]. Currently, a non-O blood group may affect hemostatic balance disturbances and lead to an increased risk of embolization compared to an O blood group [11]. By contrast, another study showed a weak relationship between $A B O$ blood type and gestational hypertension [12,13]. However, whether Rh blood type (negative or positive) is correlated to pregnancy outcomes is not known. Therefore, this study focused on the association between Rh-positive and Rh-negative blood groups and maternal pregnancy outcomes in 1,664,882 primigravida women in a large population study. The clinical characteristics did not differ significantly between Rh-positive $(n=1,661,320)$ and Rh-negative women $(n=3,290)$.

Several studies showed that the Rh blood type plays an important role in neonatal alloimmune disorders. The immune

Table 4. Multiple regression analysis of rhesus blood type as a risk factor of postpartum hemorrhage and preterm birth

\begin{tabular}{lc}
\hline Characteristics & Risk factor: adjusted OR (95\% Cl) \\
\hline Postpartum hemorrhage $^{\text {a) }}$ & $1.10(0.98-1.23)$ \\
Preterm birth $^{\text {b) }}$ & $0.02(0.85-1.23)$
\end{tabular}

$\mathrm{OR}$, odds ratio; $\mathrm{Cl}$, confidence interval.

a) Postpartum hemorrhage: adjusted for age, multiple pregnancy, cesarean section, preterm birth, low birth weight, large birth weight, preeclampsia, abruptio placenta, and placenta previa; ${ }^{\text {b) }}$ Preterm birth: adjusted for age, multiple pregnancy, and preeclampsia. 


\section{Obstetrics \& Gynecology Science}

Vol. 63, No. 4, 2020

system of a Rh-negative mother considers Rh-positive fetal cells as foreign substances. Then, the mother's body produces antibodies against fetal blood cells. Antibodies, such as $\lg \mathrm{G} 1$ and $\lg \mathrm{G} 3$, cross back through the placenta to the baby $[14,15]$. Then, they destroy the baby's circulating RBCs, and this eventually develops into complications, such as preterm delivery, LBW, hydrops fetalis, hyperbilirubinemia, anemia, and bilirubin-induced neurological dysfunction [16-20].

In this study, there was no significant association between Rh blood type and neonatal outcomes, including preterm birth, LBW, and LGA. As previously mentioned, as the risk and severity of sensitization response increases with each subsequent pregnancy, we only included primigravida women in this analysis to reduce selection bias. During the first pregnancy, the initial exposure to fetal RBCs results in the formation of IgM antibodies, and these do not cross the placental barrier. Thus, no differences were observed in the first pregnancies of Rh-negative women [21]. Based on our results, the relationship between fetal gross and Rh blood type without an alloimmune effect is weak.

The World Health Organization reports that the major contributors to maternal death or maternal mortality are postpartum hemorrhage and hypertensive disorders [22]. Various studies have investigated whether there is an association between maternal $A B O$ blood type and maternal adverse pregnancy outcomes [8,12,23-25]. In brief, maternal $A B O$ blood type was correlated to the risk of preeclampsia. However, its association with GDM, preterm delivery, LBW, and SGA remains controversial.

Compared to the ABO types, studies about Rh blood type are limited. Thus, this study evaluated the differences in the rate of cesarean section, preeclampsia, postpartum hemorrhage, abruptio placenta, placenta previa, and uterine artery embolization among pregnant women with different Rh blood types. However, there was no significant association between Rh blood type and pregnancy outcomes, even preeclampsia, in primiparous women. Lee et al. [24] showed that Rh D-positive mothers had a slightly increased risk of preeclampsia in a large cohort study in Sweden (OR, 1.07, $95 \% \mathrm{Cl}, 1.03-1.10)$. However, in this study, multiparous women were enrolled, and only the Rh D antigen was evaluated. In a previous research, more than $50 \mathrm{Rh}$ antigens have been identified at the serological level encoding 2 genes (RHD and RHCE). Among these antigens, Rh D, C, C, E, and e were considered the most clinically significant, and $R h D$ has the strongest antigenicity [26]. In our study, different findings were obtained, which might be attributed to the fact that we did not assess the differences in these antigens.

However, we did not find any associations between Rh blood type and adverse pregnancy outcomes. This result might be explained partly by the limitations of our research. First, this study only included Rh blood type classifications. However, the differences between the specific Rh blood type or the $A B O$ classification were not assessed because the $A B O$ blood group is not considered a disease category in the KNHI program. Second, as described above, the exclusion of multigravida pregnant women might have influenced the relatively varying results of the rates of preeclampsia compared to other previous studies. Third, with regard to neonatal outcomes, attention is now shifting towards shortand long-term morbidity [27]; however, in this study, only neonatal weight was included, and more detailed data are required to evaluate neonatal outcomes. To the best of our knowledge, this is the first nationwide retrospective research about the association between Rh blood type and adverse pregnancy outcomes in Korea. Hence, further studies must be conducted for a more detailed evaluation of the Rh blood type among pregnant women.

In conclusion, there were no significant differences in the risk of adverse pregnancy outcomes between Rh-positive and Rh-negative primigravida women in Korea.

\section{Acknowledgments}

This research was supported by the Basic Science Research Program through the National Research Foundation of Korea (NRF) funded by the Ministry of Science, ICT \& Future Planning (NRF-2017R1C1B2010487). This research was supported by a grant from the Korea Health Technology R\&D Project through the Korea Health Industry Development Institute (KHIDI) funded by the Ministry of Health \& Welfare, Republic of Korea (grant number: HI17C1713).

\section{Conflict of interest}

No potential conflict of interest relevant to this article was reported. 


\section{Obstetrics \& Gynecology Science}

Yihua Jin, et al. Rhesus blood type and pregnancy outcome

\section{Ethical approval}

The study protocol was approved by the institutional review board of Korea University Medical Center (IRB number: 2019GR0256).

\section{Patient consent}

This paper is a retrospective study and does not require written informed consent.

\section{References}

1. Landsteiner K, Levine P. On individual differences in human blood. J Exp Med 1928;47:757-75.

2. Wiener AS. History of the rhesus blood types. J Hist Med Allied Sci 1952;7:369-83.

3. Anstee DJ, Tanner MJ. Biochemical aspects of the blood group Rh (rhesus) antigens. Baillieres Clin Haematol 1993;6:401-22.

4. Liumbruno GM, Franchini M. Beyond immunohaematology: the role of the $A B O$ blood group in human diseases. Blood Transfus 2013;11:491-9.

5. Franchini M, Mengoli C, Lippi G. Relationship between $\mathrm{ABO}$ blood group and pregnancy complications: a systematic literature analysis. Blood Transfus 2016;14:4418.

6. Yu H, Xu N, Li ZK, Xia H, Ren HT, Li N, et al. Association of $A B O$ blood groups and risk of gastric cancer. Scand J Surg 2019:1457496919863886.

7. Paquette $M$, Dufour $R$, Baass $A$. ABO blood group is a cardiovascular risk factor in patients with familial hypercholesterolemia. J Clin Lipidol 2018;12:383-389.e1.

8. Witsenburg CP, Rosendaal FR, Middeldorp JM, Van der Meer FJ, Scherjon SA. Factor VIII levels and the risk of pre-eclampsia, HELLP syndrome, pregnancy related hypertension and severe intrauterine growth retardation. Thromb Res 2005;115:387-92.

9. Seyfizadeh N, Seyfizadeh N, Yousefi B, Borzoueisileh S, Majidinia $M$, Shanehbandi $D$, et al. Is there association between $A B O$ blood group and the risk factors of unfavorable outcomes of pregnancy? J Matern Fetal Neonatal Med 2015;28:578-82.
10. Duerbeck NB, Seeds JW. Rhesus immunization in pregnancy: a review. Obstet Gynecol Surv 1993;48:801-10.

11. Alpoim PN, de Barros Pinheiro $M$, Junqueira DR, Freitas LG, das Graças Carvalho M, Fernandes AP, et al. Preeclampsia and $\mathrm{ABO}$ blood groups: a systematic review and meta-analysis. Mol Biol Rep 2013;40:2253-61.

12. Phaloprakarn C, Tangjitgamol S. Maternal ABO blood group and adverse pregnancy outcomes. J Perinatol 2013;33:107-11.

13. Scott JR, Beer AA. Immunologic aspects of pre-eclampsia. Am J Obstet Gynecol 1976;125:418-27.

14. Finning K, Martin P, Summers J, Daniels G. Fetal genotyping for the K (Kell) and Rh C, C, and E blood groups on cell-free fetal DNA in maternal plasma. Transfusion 2007;47:2126-33.

15. Andersen AS, Praetorius L, Jørgensen HL, Lylloff K, Larsen KT. Prognostic value of screening for irregular antibodies late in pregnancy in rhesus positive women. Acta Obstet Gynecol Scand 2002;81:407-11.

16. Thompson PJ, Greenough A, Brooker R, Nicolaides KH, Gamsu HR. Antenatal diagnosis and outcome in hydrops fetalis. J Perinat Med 1993;21:63-7.

17. Kobets TV, Usachenko IE. Risk factors of complicated postnatal period in low birth weight infants. Tsitol Genet 2001;35:49-53.

18. Fan J, Lee BK, Wikman AT, Johansson S, Reilly M. Associations of rhesus and non-rhesus maternal red blood cell alloimmunization with stillbirth and preterm birth. Int J Epidemiol 2014;43:1123-31.

19. Zipursky A, Bhutani VK, Odame I. Rhesus disease: a global prevention strategy. Lancet Child Adolesc Health 2018;2:536-42.

20. Bel Hadj I, Boukhris R, Khalsi F, Namouchi M, Bougmiza I, Tinsa $F$, et al. ABO hemolytic disease of newborn : does newborn's blood group a risk factor? Tunis Med 2019;97:455-60.

21. Fyfe TM, Ritchey MJ, Taruc C, Crompton D, Galliford B, Perrin R. Appropriate provision of anti-D prophylaxis to RhD negative pregnant women: a scoping review. BMC Pregnancy Childbirth 2014;14:411.

22. Khan KS, Wojdyla D, Say L, Gülmezoglu AM, Van Look PF. WHO analysis of causes of maternal death: a systematic review. Lancet 2006;367:1066-74.

23. May D. Letter: Maternal blood group A and pre-eclampsia. BMJ 1973;4:738. 


\section{Obstetrics \& Gynecology Science}

Vol. 63, No. 4, 2020

24. Lee BK, Zhang Z, Wikman A, Lindqvist PG, Reilly M. $A B O$ and RhD blood groups and gestational hypertensive disorders: a population-based cohort study. BJOG 2012;119:1232-7.

25. Chen Y, Chen C, Ke X, Xiong L, Shi Y, Li J, et al. Analysis of circulating cholesterol levels as a mediator of an association between $A B O$ blood group and coronary heart disease. Circ Cardiovasc Genet 2014;7:43-8.
26. Chou ST, Westhoff CM. Molecular biology of the Rh system: clinical considerations for transfusion in sickle cell disease. Hematology Am Soc Hematol Educ Program 2009:178-84.

27. Smits-Wintjens VE, Walther FJ, Lopriore E. Rhesus haemolytic disease of the newborn: postnatal management, associated morbidity and long-term outcome. Semin Fetal Neonatal Med 2008;13:265-71. 